\title{
Protein Tyrosine Kinase
}

National Cancer Institute

\section{Source}

National Cancer Institute. Protein Tyrosine Kinase. NCI Thesaurus. Code C17020.

Kinases that phosphorylate protein tyrosine residues. These kinases play major roles in mitogenic signalling, and can be divided into two subfamilies: receptor tyrosine kinases, that have an extracellular ligand-binding domain, a single transmembrane domain, and an intracellular tyrosine kinase domain; and nonreceptor tyrosine kinases, which are soluble, cytoplasmic kinases. (Dictionary of Cell and Molecular Biology Online) 